\title{
The clinical features of hepatitis B virus infection and intrahepatic cholestasis in pregnancy
}

\author{
Hong Wei, Chong Zhang, Jun Meng, Zhi-Qiang Zhao, Qiu-Mei Pang \\ Beijing You'an Hospital of Capital Medical University, China
}

\begin{abstract}
Objectives: This article aimed to explore the relationship between hepatitis B virus infection (HBV) and intrahepatic cholestasis in pregnancy (ICP).

Material and methods: We conducted a retrospective study at the Beijing Youan Hospital in China between January 1, 2010 and November 31, 2016. In total, 217 pregnancies were identified and retrospectively studied. Characteristics, pregnant outcomes and the rate of mother-to-child transmission (MTCT) of HBV were compared between groups.

Results: Elevate of total bile acid occurred mainly during the second and third trimester among HBV with ICP (HBV + ICP) patients. The rate of preterm birth occurred more frequently in HBV + ICP patients than both ICP and HBV patients $(p<0.05)$. Furthermore HBV + ICP patients had a higher percentage of cesarean section, postpartum hemorrhage Apgar $<7$ at $1 / 5 \mathrm{~min}$, AFIII and LBWI rate than HBV patients $(p>0.05)$ but did not have an increased incidence of fetal loss or birth defect when compared with that in HBV and ICP patients $(p>0.05)$
\end{abstract}

Conclusions: HBV + ICP patients have adverse pregnant outcomes and as a high occurrence in the second and third trimesters of pregnancy monitoring should be enhanced at this time.

Key words: HBV; ICP; pregnancy

Ginekologia Polska 2022; 93, 5: 389-395

\section{INTRODUCTION}

Hepatitis B virus (HBV) belongs to the family hepadnaviridae and is one of the most common public health problems in the world. Hepatitis B virus infection may be as high as $2-8 \%$ in women of childbearing age in China $[1,2]$. Intrahepatic cholestasis of pregnancy (ICP) is a complication of pregnancy resulting in elevation of serum bile acid (BA), mostly occurring during the second or third trimester of pregnancy [3]. And the clinical symptoms quickly resolve after delivery $[4,5]$. Elevated BA is associated with adverse pregnancy outcomes, most notably amniotic fluid contamination, fetal asphyxia events, contributing to neonatal asphyxia, preterm birth, and sudden intrauterine fetal demise [6-8]. Cholestasis is a pathological state where bile flows from the liver into the duodenum is restricted because of dysfunctional secretion and excretion processes, leading to its release into the blood stream. Its clinical manifestations include itching, lack of power, darkened urine and jaundice. Any cause of liver cell or bile duct cell damage, or biliary tract obstruction can lead to cholestasis [9].
The pathogenesis of ICP is not fully understood. Pregnancy hormones, genetic susceptibility, the role of environmental and food factors, and changes in the gut microbiota associated with pregnancy may play a part in the development of ICP [10-12]. Estrogens and progesterone promote cholestasis by inhibiting $B A$ excretion from the hepatocyte into the bile canaliculus [13-15]. In addition, gene variants encoding hepatobiliary transporters have been identified as key factors in the development of ICP $[10,12,16]$. We noticed that, during pregnancy, many patients with HBV infection had elevated serum BA levels. However, there are few studies investigating the characteristics of patients with both HBV infection and ICP. Therefore, the clinical characteristics and pregnancy outcomes of these patients are still unclear. This article aimed to explore the relationship between HBV infection and ICP during pregnancy.

\section{Objectives}

This study aimed to examine the clinical characteristics of HBV and ICP.

\footnotetext{
Corresponding author:

Chong Zhang

e-mail:youchong89@163.com
}

Received: 30.08.2019 Accepted: 26.04.2020 Early publication date: 15.07.2021

This article is available in open access under Creative Common Attribution-Non-Commercial-No Derivatives 4.0 International (CC BY-NC-ND 4.0) license, allowing to download articles and share them with others as long as they credit the authors and the publisher, but without permission to change them in any way or use them commercially. 


\section{MATERIAL AND METHODS}

We conducted a retrospective study at the Beijing Youan Hospital in China between January 1, 2010 and November 31, 2016. This hospital is a center for diagnosing and treating hepatopathy and infectious disease during pregnancy in China. Our study was conducted with the written informed consent of the participants. The trial was approved by the institutional ethics review committee and registered with Clinical Trials.gov (no.: NCT01743079). Liver and fetal-maternal medicine specialists treated all patients regularly. All participants were followed until delivery and during the postpartum period for six weeks, and their children for at least seven months.

According to ICP diagnostic criteria, patients with itchy skin, jaundice, elevated serum BA levels, and mildly elevated liver transaminase were included [3]. HBV infection was confirmed as HBsAg positive, with a history of at least 6 months, with or without the elevation of total bilirubin and pruritus during pregnancy. Patients with both HBV infection and ICP (HBV + ICP) satisfied both the HBV and ICP standards, with an elevation of total bile acid (TBAI) during pregnancy of more than twice the normal level (> $10 \mu \mathrm{mol} / \mathrm{L}$ ). All participants meet the following criteria (Fig. 1): (I) serum alanine transaminase (ALT) and glutamic-oxaloacetic transaminase (AST) within the normal range before pregnancy, increasing to up to ten times the upper limit of the normal range (ULN, $40 \mathrm{U} / \mathrm{L}$ )) during pregnancy. ALT/AST levels returned to normal levels within 6 weeks after birth; (II) excluding twin or other multiple pregnan- cies; (III) excluding alcoholic liver disease, non-alcoholic fatty liver disease, autoimmune liver disease and other liver diseases; (IV) no pre-existing chronic conditions, such as diabetes, high blood pressure or heart disease; $(V)$ absence of other infectious diseases such as human immunodeficiency virus, active syphilis or hepatitis $C$ virus infection; ( $\mathrm{VI})$ absence of immunoglobulin $\mathrm{M}$ antibodies against toxoplasma, rubella virus, cytomegalovirus, herpes simplex virus. Patients who had incomplete pregnancy data or whose pregnancy was terminated before 12 weeks were excluded.

A total of 217 pregnant women were identified and reviewed. Of these, 99 patients were diagnosed with both HBV infection and ICP (Group I), 19 had ICP without HBV infection (Group II) and 99 had HBV infection without ICP (Group III). Characteristics, pregnancy outcomes and the rate of mother-to-child transmission (MTCT) of HBV were compared between groups.

Adverse maternal outcomes were pregnancy-induced hypertension (PIH), including gestational hypertension and preeclampsia, gestational diabetes mellitus (GDM), premature rupture of membrane (PROM), preterm birth $(<34 \mathrm{w}$ and $<32 \mathrm{w}$ ) and postpartum hemorrhage. Adverse fetal outcomes were fetal loss (including late abortion, intrauterine death, induced labor and perinatal death), birth defects, low birth weight infant ( $\mathrm{LBWl},<2,500 \mathrm{~g}$ ), Apgar score ( $<7$ at 1 or $5 \mathrm{~min}$ ) and amniotic fluid III degrees contamination (AFIII, the amniotic fluid is yellowish-green or brown with a large number of feces).

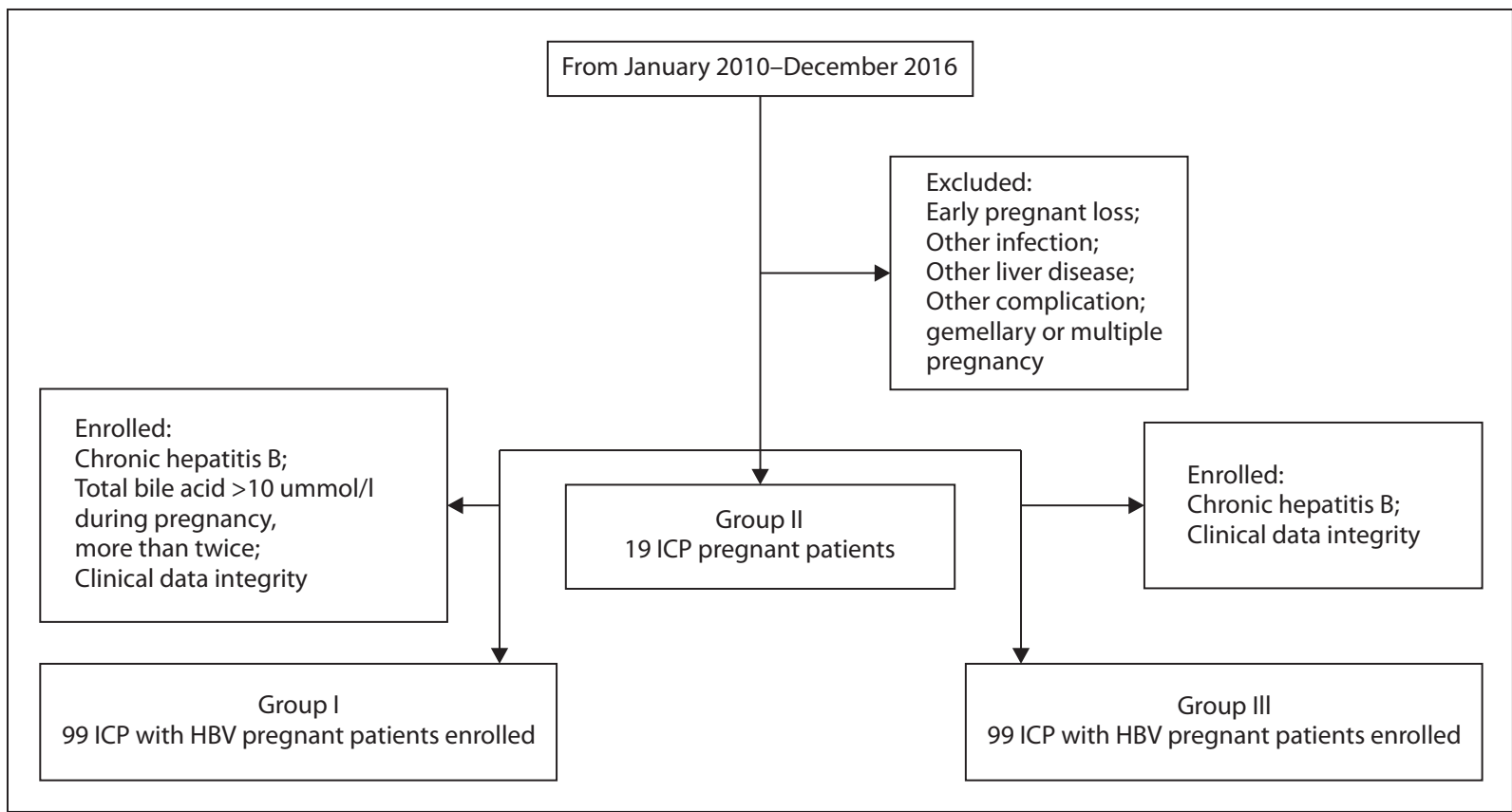

Figure 1. Flowchart of patients' enrollment 
Serum HBsAg and hepatitis B e antigen levels were determined using reagents from Roche Diagnostics and a Cobas e601 analyzer (Roche Diagnostic GmbH, Mannheim, Germany). HBV DNA was quantitatively measured using a real-time PCR assay (Sansure Biotech Inc., Changsha, Hunan, China). Infants of all mothers received $200 \mathrm{IU}$ of hepatitis B immunoglobulin (HBIG) and 10mg of HBV vaccine within 6 hours of birth, then 200 IU of HBIG at 21 days of age, and two more vaccinations were given at 1 month and 6 months of age. Serum HBV-DNA and HBV markers were measured at birth and 7 months of age. Intrauterine infection was defined as peripheral blood HBV-DNA/HBsAg positive at birth and continued positive at 7 months. Intrahepatic $\mathrm{HBV}$ infection was defined as the transient presence of HBV-DNA/HBsAg after delivery. MTCT of HBV includes both intrauterine infection and postpartum contamination, which was defined as peripheral blood HBV-DNA/HBsAg was negative at birth and peripheral blood HBV-DNA/HBsAg was positive at 7 months of age.

The continuous data were expressed as mean \pm standard deviation (SD), and the classified data were expressed as percentages. The $x 2$ test or Fisher's exact test were used to classify variables. All analyses were performed using SPSS version 20 (IBM, Armonk, NY, USA). When $p<0.05$, the difference was considered statistically significant.

\section{RESULTS}

The demographic and characteristic data are summarized in Table 1. Most patients were uniparous and the difference between groups was not statistically significant.
The average age in Group I was $28.97 \pm 3.93$ years. Elevated TBAI occurred mainly during the second and third trimester in both Group I and II. In Group I the rate of elevated TBAI in the second trimester was $40.4 \%$ which was higher than that in Group II and 51.5\% in third trimester which was lower than that in Group II. The incidence of mild and severe TBAI elevation in patients in Group I was $52.53 \%$ and $47.47 \%$, respectively, and neither was significantly different from Group II. The proportion of live births in Group I was 94.95\%, which was not significantly different from Group II or III. In Group I, $34.34 \%$ were delivered vaginally and $60.61 \%$ by cesarean section. More vaginal deliveries and fewer cesarean deliveries were recorded in Group III compared with Group I (both $p<0.05$ ). However, there was no significant difference in delivery mode between Group I and Group II.

Table 2 shows the pregnancy outcomes of all patients. The incidence of preterm birth in Group I was 24.24\%, higher than that in Group II and III. It was noted that in the 24 cases of preterm birth there were 4 births at $<32 \mathrm{w}$ and 7 at $<34$ w. No cases at $<32$ w occurred in either Group II or III. However, there was no significant difference in the frequency of preterm births between Groups I and II, or between Groups I and III. The incidence of postpartum hemorrhage in Group I was $11.11 \%$, higher than that in Group III, but not significantly different from that in Group II. In addition, there was no significant difference between groups for other complications such as PIH, GDM and PROM.

Several differences in fetal outcomes were observed between Group I and Group III: a higher percentage of patients in Group I suffered from an Apgar score $<7$ at $1 \mathrm{~min}$

\begin{tabular}{|c|c|c|c|c|c|c|c|c|}
\hline \multirow[t]{3}{*}{ Characteristics } & \multicolumn{2}{|c|}{ Group I } & \multicolumn{2}{|c|}{ Group II } & \multicolumn{2}{|c|}{ Group III } & P1 & P2 \\
\hline & \multicolumn{2}{|c|}{$n=99$} & \multicolumn{2}{|c|}{$n=19$} & \multicolumn{2}{|c|}{$n=99$} & & \\
\hline & $\mathbf{n}$ & $\%$ & $\mathbf{n}$ & $\%$ & $\mathbf{n}$ & $\%$ & & \\
\hline Unipara & 71 & 71.72 & 17 & 89.47 & 60 & 57.58 & 0.18 & 0.037 \\
\hline Multipara & 28 & 28.28 & 2 & 10.53 & 39 & 42.42 & 0.18 & 0.037 \\
\hline Age [Y] & \multicolumn{2}{|c|}{$28.97 \pm 3.93$} & \multicolumn{2}{|c|}{$28.21 \pm 5.64$} & \multicolumn{2}{|c|}{$29.23 \pm 3.72$} & & \\
\hline \multicolumn{9}{|l|}{ GWTBA [W] } \\
\hline First trimester & 8 & 8.10 & 0 & 0 & & & & \\
\hline Second & 40 & 40.40 & 2 & 10.53 & & & 0.013 & \\
\hline Third & 51 & 51.52 & 17 & 89.47 & & & 0.002 & \\
\hline \multicolumn{9}{|l|}{ DBA } \\
\hline Mild & 52 & 52.53 & 7 & 36.84 & & & 0.21 & \\
\hline Severe & 47 & 47.47 & 12 & 63.16 & & & 0.21 & \\
\hline Live birth & 94 & 94.95 & 18 & 94.74 & 98 & 98.99 & 1 & 0.214 \\
\hline by vagina & 34 & 34.34 & 5 & 26.32 & 56 & 56.57 & 0.496 & $<0.01$ \\
\hline by cesarean section & 60 & 60.61 & 13 & 68.42 & 42 & 42.42 & 0.521 & 0.01 \\
\hline
\end{tabular}

GWTBA — gestational weeks of rising of total bile acids; DBA — degree of bile acid increase; P1 — Group I vs Group II; P2 — Group I vs Group III 


\begin{tabular}{|c|c|c|c|c|c|c|c|c|}
\hline \multirow[t]{3}{*}{ Pregnancy outcome } & \multirow{2}{*}{\multicolumn{2}{|c|}{$\begin{array}{l}\text { Group I } \\
n=99\end{array}$}} & \multirow{2}{*}{\multicolumn{2}{|c|}{$\begin{array}{c}\text { Group II } \\
n=19\end{array}$}} & \multirow{2}{*}{\multicolumn{2}{|c|}{$\begin{array}{c}\text { Group III } \\
n=99\end{array}$}} & \multirow[t]{3}{*}{ P1 } & \multirow[t]{3}{*}{ P2 } \\
\hline & & & & & & & & \\
\hline & $n$ & $\%$ & $n$ & $\%$ & n & $\%$ & & \\
\hline \multicolumn{9}{|l|}{ Maternal } \\
\hline $\mathrm{PIH}$ & 7 & 7.07 & 2 & 10.52 & 3 & 3.03 & 0.962 & 0.194 \\
\hline Gestational hypertension & 2 & 2.02 & 0 & 0 & 2 & 2.02 & & 1 \\
\hline Preeclampsia & 5 & 5.05 & 2 & 10.52 & 1 & 1.01 & 0.693 & 0.214 \\
\hline GDM & 20 & 20.20 & 5 & 26.32 & 13 & 13.13 & 0.771 & 0.182 \\
\hline PROM & 15 & 15.15 & 3 & 15.79 & 13 & 13.13 & 1 & 0.683 \\
\hline 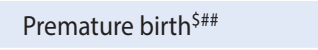 & 24 & 24.24 & 9 & 47.37 & 2 & 2.02 & 0.04 & $<0.01$ \\
\hline$<32 w$ & 4 & 4.04 & 0 & 0 & 0 & 0 & & \\
\hline$<34 w$ & 7 & 7.07 & 3 & 15.79 & 2 & 2.02 & 0.424 & 0.172 \\
\hline Postpartum hemorrhage ${ }^{\# \#}$ & 11 & 11.11 & 2 & 10.53 & 2 & 2.02 & 1 & 0.01 \\
\hline \multicolumn{9}{|l|}{ Fetal } \\
\hline Fetal loss & 5 & 5.05 & 2 & 10.52 & 1 & 1.01 & 0.693 & 0.214 \\
\hline Late abortion & 1 & 1.01 & 0 & 0 & 0 & 0 & & \\
\hline Intrauterine death & 2 & 2.02 & 0 & 0 & 1 & 1.01 & & 1 \\
\hline Induced labor & 1 & 1.01 & 1 & 5.26 & 0 & 0 & 0.73 & \\
\hline Perinatal death & 1 & 1.01 & 1 & 5.26 & 0 & 0 & 0.73 & \\
\hline Apgar $<7$ at $1 \mathrm{~min}^{\# \#}$ & 30 & 30.30 & 4 & 21.05 & 6 & 6.06 & 0.415 & $<0.01$ \\
\hline Apgar $<7$ at $5 \mathrm{~min}^{\# \#}$ & 25 & 25.25 & 4 & 21.05 & 4 & 4.04 & 0.921 & $<0.01$ \\
\hline AFIII"\#\# & 31 & 31.31 & 10 & 52.63 & 12 & 12.12 & 0.074 & $<0.01$ \\
\hline Birth defects & 7 & 7.07 & 1 & 5.26 & 4 & 4.04 & 1 & 0.352 \\
\hline LBWI"\# & 15 & 15.15 & 7 & 36.84 & 2 & 2.02 & 0.057 & $<0.01$ \\
\hline
\end{tabular}

AFIII - degree of contamination of the amniotic fluid reaching III degrees; P1 - Group I vs Group II; P2 — Group I vs Group III; $\$$ - Group I > Group II, P1 < 0.5 ; \#\# — Group I $>$ Group III, P2 < 0.01

(30.30\% vs 6.06\%, p < 0.01), Apgar $<7$ at 5 min $(25.25 \%$ vs $4.04 \%, p<0.01)$, AFIII $(31.31 \%$ vs $12.12 \%, p<0.01)$ and LBWI $(15.15 \%$ vs $2.02 \%, p<0.01)$. The incidence of these adverse infant outcomes was not significantly different between Group I and Group II. Five cases suffered from fetal loss in Group I, including 1 case of late abortion, 2 cases of intrauterine death, 1 case of induced labor and 1 case of perinatal death. Notably, Group I did not have an increased incidence of fetal loss compared with Groups II and III. No difference in frequency of other adverse fetal outcomes, such as birth defects, was observed between Group I and II or Group I and III.

Rates of HBV infection of infants in Group I and III was shown in Table 3. Ninety-nine pregnancies in Group I and III resulted in 94 and 98 live births, respectively. In Group I, only four infants from birth to 7 months were HBsAg and/or HBV-DNA positive. Eight infants in Group I were born HBsAg and/or HBV-DNA positive but turned negative at 7 months (intrapartum contamination). One infant in Group I was postpartum contamination. In Group III, eight babies were born HBsAg and/or HBV-DNA positive, and six of the babies were HBsAg negative at 7 months of age. In addition, one child in Group III was born HBsAg and HBV-DNA negative but became positive at 7 months. Thus, the infection rates of intrauterine HBV were $4.26 \%$ and $2.04 \%$ in Group I and Group III, while the rates of MTCT of HBV were 5.32\% and 3.06\% respectively. Despite Group I showing a trend towards higher occurrence of intrauterine HBV infection and MTCT of HBV, there were no statistically significant differences between the two groups.

\section{DISCUSSION}

The reason patients with chronic HBV develop cholestasis in pregnancy is still unclear. It may be that HBV infection causes an autoimmune response that eventually leads to liver cell damage. Moreover, it is known that gestational hormone level changes like estrogens and progesterone during pregnancy aggravate liver disease by placing extra burden on the liver $[7,17,18]$. Both liver cell damage and gestational hormone level changes can inhibit BA excretion from the hepatocyte into the bile canaliculus and ultimately lead to cholestasis. 


\begin{tabular}{|c|c|c|c|c|c|}
\hline \multirow{3}{*}{ HBV Infection of Infants } & \multirow{2}{*}{\multicolumn{2}{|c|}{$\begin{array}{c}\text { Group I } \\
n=94\end{array}$}} & \multirow{2}{*}{\multicolumn{2}{|c|}{$\begin{array}{c}\text { Group III } \\
n=98\end{array}$}} & \multirow{3}{*}{ P1 } \\
\hline & & & & & \\
\hline & $\mathbf{n}$ & $\%$ & $\mathbf{n}$ & $\%$ & \\
\hline \multicolumn{6}{|l|}{ At birth } \\
\hline HBsAg + HBVDNA + & 8 & 8.51 & 5 & 5.10 & 0.347 \\
\hline HBsAg + HBVDNA - & 4 & 4.26 & 3 & 3.06 & 0.955 \\
\hline HBsAg - HBVDNA - & 82 & 87.23 & 90 & 91.84 & 0.297 \\
\hline \multicolumn{6}{|l|}{ After 7 month } \\
\hline HBsAg + HBVDNA + & 3 & 3.19 & 1 & 1.02 & 0.584 \\
\hline HBsAg + HBVDNA - & 2 & 2.13 & 2 & 2.04 & 1 \\
\hline HBsAg - HBVDNA - & 4 & 4.26 & 6 & 6.12 & 0.797 \\
\hline $\mathrm{HBsAb+}$ & 85 & 90.42 & 89 & 90.81 & 0.926 \\
\hline Intrauterine infection & 4 & 4.26 & 2 & 2.04 & 0.641 \\
\hline Intrapartum contamination & 1 & 1.06 & 1 & 1.02 & 1 \\
\hline Postpartum contamination & 1 & 1.06 & 1 & 1.02 & 1 \\
\hline MTCT of HBV & 5 & 5.32 & 3 & 3.06 & 0.673 \\
\hline
\end{tabular}

P1 - Group I vs Group III

Many studies have reported on the pregnancy outcomes of patients with ICP. Most of these studies have considered ICP as a transient benign symptom in mothers resulting primarily in preterm birth (spontaneous and iatrogenic) [19-22]. Recently, ICP has been reported to be associated with pre-eclampsia and GDM [19, 21]. But for babies, it can have serious consequences, most notably amniotic fluid contamination, fetal asphyxia events, contributing to neonatal asphyxia and sudden intrauterine fetal demise $[3,6,8,19-21]$. The mechanism underlying the pathogenesis of ICP and the mechanisms by which ICP leads to poor fetal outcome are unclear. Possible evidence comes from in vitro and laboratory animal studies. In animal studies, BA has been shown to stimulate gut motility. Injection of BA into pregnant sheep increased the preterm birth rate, and in every case feces were detected in the amniotic fluid [23]. The study seems to show that ICP can lead to amniotic fluid contamination and premature birth. As for the mechanism by which ICP leads to acute hypoxia and fetal death, it may induce vasoconstriction of chorionic veins [24], oxidative stress in the placenta and increased apoptosis in the fetal liver [25], or trigger fetal cardiac arrhythmias that lead to cardiac arrest [26, 27].

The only one study that investigated pregnancy outcomes of patients with ICP and HBV infection, mainly focused on fetal outcomes [28]. In our study, we studied both maternal and fetal outcomes of patients with both HBV infection and ICP (Group I) by comparing them with patients diagnosed with either ICP alone (Group II) or HBV infection alone (Group III) during pregnancy. We found that like ICP patients, elevated TBAI occurred mainly during the second and third trimester in pregnancy among HBV + ICP patients. Although there was no significant difference in live birth rates between these groups, HBV + ICP patients had a higher percentage of CS than HBV patients. Notably our study showed no increase in the CS rate between HBV+ICP and ICP patients, which was not consistent with Hu's study [28]. Likewise, we did not find a difference in the occurrence of maternal outcomes such as PIH, GDM and PROM; this was the first report of the study of these outcomes. Interestingly, we found that preterm birth occurred more frequently in $\mathrm{HBV}+\mathrm{ICP}$ patients than either the ICP or HBV patients which was consistent with Hu's study. Furthermore, HBV + ICP patients had a higher postpartum hemorrhage rate than HBV patients but not the ICP group. A possible explanation is that chronic hepatitis causes liver damage, which is the site for the synthesis of various clotting factors and cholestasis can account for steatorrhea which can affect the absorption of vitamin K, and eventually lead to hemorrhage [29-31]. However, the exact mechanism has not been reported.

We also found that adverse fetal outcomes were increased in HBV + ICP patients, with higher rates of Apgar score $<7$ at 1 and $5 \mathrm{~min}$, AFIII and LBWI than HBV patients, which was consistent with Hu's study [28]. There was no difference in the incidence of adverse fetal outcomes compared with ICP patients. This finding was inconsistent with the previous study [28]. However, HBV+ICP patients did not have an increased incidence of fetal loss or birth defects when compared with HBV or ICP patients. Therefore, we believe that the adverse fetal outcome of patients with HBV + ICP 
is the same as that of patients with ICP and HBV + ICP patients had more adverse fetal outcomes than HBV patients. The possible reason may be that in HBV + ICP patients, cholestasis may cause fetal hypoxia and distress such as low Apgar score ( $<7$ at 1 and $5 \mathrm{~min}$ ) and AFIII by the same mechanism that ICP causes adverse fetal outcomes [24-27]. Patients with HBV+ICP may develop chorionic vascular lesions in the placenta, resulting in placental dysfunction, aggravated cholestasis, fetal hypoxia, and eventually LBWI [32]. Moreover, the higher rate of preterm birth in HBV + ICP patients than either the ICP or HBV patients would also lead to LBWI. The higher rate of adverse maternal and fetal outcomes in $\mathrm{HBV}+\mathrm{ICP}$ patients than in HBV patients may account for the higher CS rate, as fetal hypoxia and distress occurred more frequently. These findings suggest that HBV + ICP patients have a higher frequency of adverse outcomes in the second and third trimesters of pregnancy, therefore monitoring should be enhanced during this period.

Notably, our study reported the MTCT of HBV in $\mathrm{HBV}+\mathrm{ICP}$ patients and compared it with HBV patients during pregnancy. MTCT of HBV was found to be a primary reason for vaccination failure, therefore it is important to know whether cholestasis enhances the rate of MTCT of HBV. Hu's study suggested that cholestasis would increase the rate of MTCT of HBV. Our results also showed a tendency toward a higher rate of MTCT of HBV in HBV + ICP patients, although it was not statistically significantly increased over HBV patients. This finding might indicate that cholestasis can increase the vaccination failure rate in HBV patients. However, further study is required to confirm this.

Our study has several limitations. First, our single-center study may be biased. Our hospital is a tertiary hospital in China that accepts liver disease patients from other community hospitals. So, liver abnormalities may be common in patients during pregnancy, which may not be representative of the general population, and we do not have a healthy group as our control group. Second, we examined patients who have elevated $B A$, without grouping according to the degree of BA increase as we studied a relatively small sample of ICP patients. Third, our study measured adverse fetal outcomes in HBV + ICP patients, but we did not follow up the children for a long time. Therefore, we do not know whether these results will lead to more serious long-term effects.

\section{CONCLUSIONS}

In summary, our study found that patients with ICP and HBV infection have adverse pregnancy outcomes, with a high occurrence in the second and third trimesters of pregnancy, therefore monitoring should be enhanced, and active treatment should be undertaken at this time. More studies focusing on characteristics of patients with both $\mathrm{HBV}$ infection and ICP are needed in the future.

\section{Conflict of interest}

All authors declare no conflict of interest.

\section{REFERENCES}

1. Huang $Y$, Li L, Sun $X$, et al. Screening of pregnant women for hepatitis $B$ virus surface antigen ( $\mathrm{HBsAg}$ ) and subsequent management, Qiandongnan prefecture, Guizhou, China, 2010. Vaccine. 2013; 31(Suppl 9): J62-J65, doi: 10.1016/j.vaccine.2013.05.103, indexed in Pubmed: 24331022.

2. Lao TT, Sahota DS, Law LW, et al. Age-specific prevalence of hepatitis B virus infection in young pregnant women, Hong Kong Special Administrative Region of China. Bull World Health Organ. 2014; 92(11): 782-789, doi: 10.2471/BLT.13.133413, indexed in Pubmed: 25378739.

3. Geenes V, Lövgren-Sandblom A, Benthin L, et al. The reversed feto-maternal bile acid gradient in intrahepatic cholestasis of pregnancy is corrected by ursodeoxycholic acid. PLoS One. 2014; 9(1): e83828, doi: 10.1371/journal.pone.0083828, indexed in Pubmed: 24421907.

4. Medina Lomeli JM, Jauregui Melendrez RA, Medina Castro N, et al. Intrahepatic cholestasis of pregnancy: review [article in Spanish]. Ginecol Obstet Mex. 2012; 80(4): 285-294, indexed in Pubmed: 22808859.

5. Turunen K, Mölsä A, Helander K, et al. Health history after intrahepatic cholestasis of pregnancy. Acta Obstet Gynecol Scand. 2012; 91(6): 679-685, doi: 10.1111/j.1600-0412.2012.01403.x, indexed in Pubmed: 22458935.

6. Martinefski M, Contin M, Samassa P, et al. The importance of analytical methodology in accurate diagnosis and monitoring of intrahepatic cholestasis of pregnancy. Int J Gynaecol Obstet. 2013; 123(1): 78-79, doi: 10.1016/j.ijgo.2013.06.012, indexed in Pubmed: 23915527.

7. Turunen K, Helander K, Mattila K, et al. Menopause after a history of intrahepatic cholestasis of pregnancy. Menopause. 2013; 20(11): 1200-1203, doi: 10.1097/gme.0000000000000101, indexed in Pubmed: 24149924.

8. Wikström Shemer E, Thorsell M, Östlund E, et al. Stereological assessment of placental morphology in intrahepatic cholestasis of pregnancy. Placenta. 2012; 33(11): 914-918, doi: 10.1016/j.placenta.2012.08.005, indexed in Pubmed: 23020907.

9. European Association for the Study of the Liver. EASL clinical practice guidelines: management of cholestatic liver diseases. J Hepatol. 2009; 51(2): 237-267, doi: 10.1016/j.jhep.2009.04.009, indexed in Pubmed: 19501929.

10. Lammert F, Marschall HU, Glantz A, et al. Intrahepatic cholestasis of pregnancy: molecular pathogenesis, diagnosis and management. J Hepatol. 2000; 33(6): 1012-1021, doi: 10.1016/s0168-8278(00)80139-7, indexed in Pubmed: 11131439.

11. Reyes H. Sex hormones and bile acids in intrahepatic cholestasis of pregnancy. Hepatology. 2008; 47(2): 376-379, doi: 10.1002/hep.22139.

12. Dixon PH, Williamson C. The pathophysiology of intrahepatic cholestasis of pregnancy. Clin Res Hepatol Gastroenterol. 2016; 40(2): 141-153, doi: 10.1016/j.clinre.2015.12.008, indexed in Pubmed: 26823041.

13. Lee JM, Trauner M, Soroka CJ, et al. Expression of the bile salt export pump is maintained after chronic cholestasis in the rat. Gastroenterology. 2000; 118(1): 163-172, doi: 10.1016/s0016-5085(00)70425-2, indexed in Pubmed: 10611165.

14. Vallejo M, Briz O, Serrano MA, et al. Potential role of trans-inhibition of the bile salt export pump by progesterone metabolites in the etiopathogenesis of intrahepatic cholestasis of pregnancy. J Hepatol. 2006; 44(6): 1150- 1157, doi: 10.1016/j.jhep.2005.09.017, indexed in Pubmed: 16458994.

15. Abu-Hayyeh S, Martinez-Becerra P, Sheikh Abdul Kadir SH, et al. Inhibition of $\mathrm{Na}$--taurocholate $\mathrm{Co}$-transporting polypeptide-mediated bile acid transport by cholestatic sulfated progesterone metabolites. J Biol Chem. 2010; 285(22): 16504-16512, doi: 10.1074/jbc.M109.072140, indexed in Pubmed: 20177056.

16. Keitel V, Dröge C, Stepanow S, et al. Intrahepatic cholestasis of pregnancy (ICP): case report and review of the literature. Z Gastroenterol. 2016; 54(12): 1327-1333, doi: 10.1055/s-0042-118388, indexed in Pubmed: 27936482.

17. Floreani A, Caroli D, Lazzari R, et al. Intrahepatic cholestasis of pregnancy: new insights into its pathogenesis. J Matern Fetal Neonatal Med. 2013; 26(14): 1410-1415, doi: 10.3109/14767058.2013.783810, indexed in Pubmed: 23480690 .

18. Zhou F, He MM, Liu ZF, et al. Expression of corticotrophin-releasing hormone and its receptor in patients with intrahepatic cholestasis of pregnancy. Placenta. 2013; 34(5): 401-406, doi: 10.1016/j.placenta.2013.02.006, indexed in Pubmed: 23478074. 
19. Arrese $M$, Reyes $\mathrm{H}$. Intrahepatic cholestasis of pregnancy: a past and present riddle. Ann Hepatol. 2006; 5(3): 202-205, indexed in Pubmed: 17060884.

20. Wikström Shemer $\mathrm{E}$, Marschall HU, Ludvigsson JF, et al. Intrahepatic cholestasis of pregnancy and associated adverse pregnancy and fetal outcomes: a 12-year population-based cohort study. BJOG. 2013; 120(6): 717-723, doi: 10.1111/1471-0528.12174, indexed in Pubmed: 23418899.

21. Glantz A, Marschall HU, Mattsson LA. Intrahepatic cholestasis of pregnancy: Relationships between bile acid levels and fetal complication rates. Hepatology. 2004; 40(2): 467-474, doi: 10.1002/hep.20336, indexed in Pubmed: 15368452.

22. Germain AM, Kato S, Carvajal JA, et al. Bile acids increase response and expression of human myometrial oxytocin receptor. Am J Obstet Gynecol. 2003; 189(2): 577-582, doi: 10.1067/s0002-9378(03)00545-3, indexed in Pubmed: 14520238.

23. Campos GA, Guerra FA, Israel EJ. Effects of cholic acid infusion in fetal lambs. Acta Obstet Gynecol Scand. 1986; 65(1): 23-26, doi: 10.3109/00016348609158224, indexed in Pubmed: 3716776.

24. Sepúlveda WH, González C, Cruz MA, et al. Vasoconstrictive effect of bile acids on isolated human placental chorionic veins. Eur J Obstet Gynecol Reprod Biol. 1991; 42(3): 211-215, doi: 10.1016/0028-2243(91)90222-7, indexed in Pubmed: 1773876.

25. Perez MJ, Macias RIR, Duran C, et al. Oxidative stress and apoptosis in fetal rat liver induced by maternal cholestasis. Protective effect of ursodeoxycholic acid. J Hepatol. 2005; 43(2): 324-332, doi: 10.1016/j. jhep.2005.02.028, indexed in Pubmed: 15970352.
26. Williamson C, Gorelik J, Eaton BM, et al. The bile acid taurocholate impairs rat cardiomyocyte function: a proposed mechanism for intra-uterine fetal death in obstetric cholestasis. Clin Sci (Lond). 2001; 100(4):363-369, indexed in Pubmed: 11256973.

27. Sheikh Abdul Kadir SH, Miragoli M, Abu-Hayyeh S, et al. Bile acid-induced arrhythmia is mediated by muscarinic $\mathrm{M} 2$ receptors in neonatal rat cardiomyocytes. PLoS One. 2010; 5(3): e9689, doi: 10.1371/journal. pone.0009689, indexed in Pubmed: 20300620.

28. Hu Y, Ding YL, Yu L. The impact of intrahepatic cholestasis of pregnancy with hepatitis B virus infection on perinatal outcomes. Ther Clin Risk Manag. 2014; 10: 381-385, doi: 10.2147/TCRM.S61530, indexed in Pubmed: 24920912.

29. Geenes V, Williamson C. Intrahepatic cholestasis of pregnancy. World J Gastroenterol. 2009; 15(17): 2049-2066, doi: 10.3748/wjg.15.2049, indexed in Pubmed: 19418576.

30. Lammert F, Marschall HU, Matern S. Intrahepatic cholestasis of pregnancy. Curr Treat Options Gastroenterol. 2003; 6(2): 123-132, doi: 10.1007/s11938-003-0013-x, indexed in Pubmed: 12628071.

31. Marschall HU. Management of intrahepatic cholestasis of pregnancy. Expert Rev Gastroenterol Hepatol. 2015; 9(10): 1273-1279, doi: 10.1586/17474124.2015.1083857, indexed in Pubmed: 26313609.

32. Chen LZ, Zhou WQ, Zhao SS, et al. A nested case-control study of maternal-neonatal transmission of hepatitis B virus in a Chinese population. World J Gastroenterol. 2011; 17(31): 3640-3644, doi: 10.3748/wjg. v17.i31.3640, indexed in Pubmed: 21987612. 